\title{
Optimal Design of a Three-Phase AFPM for In- Wheel Electrical Traction
}

\author{
Harold Saavedra, Luís Romeral \\ Electronic Engineering Department \\ Universitat Politècnica de Catalunya \\ Terrassa, Spain \\ Harold.saavedra@mcia.upc.edu, romeral@eel.upc.edu
}

\author{
Jordi-Roger Riba \\ Electric Engineering Department \\ Universitat Politècnica de Catalunya \\ Terrassa, Spain \\ riba@ee.upc.edu
}

\begin{abstract}
Sinusoidally fed permanent magnet synchronous motors (PMSM) fulfill the special features required for traction motors to be applied in electric vehicles (EV). Among them, axial flux permanent magnet (AFPM) synchronous motors are especially suited for in-wheel applications. Electric motors used in such applications must meet two main requirements, i.e. high power density and fault tolerance. This paper deals with the optimal design of an AFPM for in-wheel applications used to drive an electrical scooter. The single-objective optimization process carried out in this paper is based on designing the AFPM to obtain an optimized power density while ensuring appropriate fault tolerance requirements. For this purpose a set of analytical equations are applied to obtain the geometrical, electric and mechanical parameters of the optimized AFPM and several design restrictions are applied to ensure fault tolerance capability. The optimization process is based on a genetic algorithm and two more constrained nonlinear optimization algorithms in which the objective function is the power density. Comparisons with available data found in the technical bibliography show the appropriateness of the approach developed in this work.
\end{abstract}

Keywords-Permanent magnet; fault tolerance; optimization;genetic algorithm.

\section{NOMENCLATURE}

$P_{R}: \quad$ Rated power

$m: \quad$ Number of phases

$m_{l}$ : $\quad$ Phases per stator. It is unity in the case of one stator

$p: \quad$ Number of pole pairs

$f: \quad$ Electric frequency

$Q: \quad$ Number of slots in the stator

$K_{e}$ : $\quad E M F$ factor incorporating the winding distribution factor and the radio between the area spanned by the salient poles and the total air gap area

$K_{i}: \quad$ Form factor of the electric current wave $=I_{p k} / I_{R M S}$

$K_{p}: \quad$ Form factor of the electric power wave

$K_{c u}: \quad$ Slot fill factor

$K_{c}: \quad$ Carter coefficient

$\eta$ : $\quad$ Overall machine efficiency

$B_{g}$ : $\quad$ Air gap flux density

$B_{c s}$ : $\quad$ Stator core flux density

$A_{s}: \quad$ Stator electrical loading

$f_{b}$ : $\quad$ Rated or base converter frequency $\omega_{b}: \quad$ Base angular electrical frequency $=2 \pi f_{b}$

$p$ : $\quad$ Pole pairs number

$N_{p h}$ : Number of turns per phase

$L_{e}: \quad$ Effective stack length

$L_{r}: \quad$ Rotor axial length

$L_{c r}: \quad$ Rotor core axial length

$L_{s}$ : $\quad$ Stator axial length

$L_{c s}: \quad$ Stator core axial length

$L_{P M}$ : $\quad$ Permanent magnets axial length

g: $\quad$ Air gap length

$B_{r}: \quad$ Remanent flux density of the permanent magnets

$B_{u}$ : Attainable flux density on the surface of the permanent magnets

$B_{c r}: \quad$ Flux density in the rotor core

$B_{c s}: \quad$ Flux density in the stator core

$J_{s}: \quad$ Current density

$\mu_{r, P M}: \quad$ Relative permeability of the permanent magnets

$K_{d}: \quad$ Flux leakage factor

$W_{\text {cuo }}$ : Outer protrusion in the radial direction of the end winding

$W_{c u i}$ : $\quad$ Inner protrusion in the radial direction of the end winding

$D_{o}$ : $\quad$ Outer surface diameter of the AFPM

$D_{t}$ : $\quad$ Total outer diameter

$D_{g}$ : $\quad$ Average diameter

$D_{i}$ : $\quad$ Inner diameter

$K_{l}$ : $\quad$ Aspect ratio coefficient $=D_{g} / L_{e}$

$\lambda$ : $\quad$ Ratio between the inner and outer diameters $=D_{i} / D_{o}$

$I_{S, N}: \quad$ Rated stator current

$V_{p h, N}: \quad$ Rated phase-to-neutral voltage

$\psi_{N}$ : $\quad$ Nominal flux linkage

\section{INTRODUCTION}

Electric vehicles (EVs) are attractive due to several benefits compared to internal combustion engine powered vehicles, including low emissions, higher efficiency, quieter operation, less maintenance costs, stronger acceleration or lower fuel price, among others. However, their main drawbacks are associated to batteries, which have a limited driving range, long recharge time and high cost [1].

This work was supported by the Catalan Agència de Gestió d'Ajuts Universitaris i de Recerca under the AGAUR 2014 SGR 101 Research Project. 
In part due to the limitations of the battery pack, EVs require traction motors with especial features, including high efficiency [2], high power and torque density, compactness, precise torque control, extended speed range $[3,4]$. Sinusoidally fed PMSMs are also compatible with adjustable speed ac inverter drives since they are compatible with advanced control techniques thus transferring them of enhanced controllability when compared to rectangular-fed brushless dc permanent magnet motors (BLDC) [5]. Due to the abovementioned appealing features, sinusoidally fed PMSMs are being applied as traction motors in EVs.

AFPM synchronous motors are disc-shaped and very compact, therefore being a valid solution for direct drive inwheel applications [6]. The direct drive motor allows simplifying the mechanical structure of the vehicle, thus increasing overall efficiency and minimizing vehicle weight. AFPMs also show an inherent anti-lock braking system (ABS) capability since they can generate a negative torque [7]. In addition, kinetic energy can be recovered during braking stages by applying a regenerative braking strategy. Due to their disclike shape, AFPMs allow different designs including multiple or single air gaps, with slots or slot less configurations or ironless designs [8]. These types of machines are often classified into internal stator (torus) and internal rotor topologies, depending on the function of the central disk. The torus topology includes both $\mathrm{NN}$ and NS configurations, where NS refers to a north pole $\mathrm{N}$ facing a south pole $\mathrm{S}$ placed at the other side of the stator [7].

This paper deals with the internal stator or torus topology, which has two outer rotor discs in a NN configuration. The axially magnetized permanent magnets are glued to both inner faces of the rotor discs. The stator is made of strip wound steel and contains concentrated back-to-back connected windings wounded inside the slots [8]. These radial windings, which are wounded in the radial direction, are required to produce the electromagnetic torque. Due to the multiple air gaps configuration, the use of back-to-back windings allow minimizing copper losses due to the reduced end windings length when compared with other configurations, thus enhancing overall efficiency [3].

Fig. 1 shows a dual outer rotor AFPM topology with NN configuration.

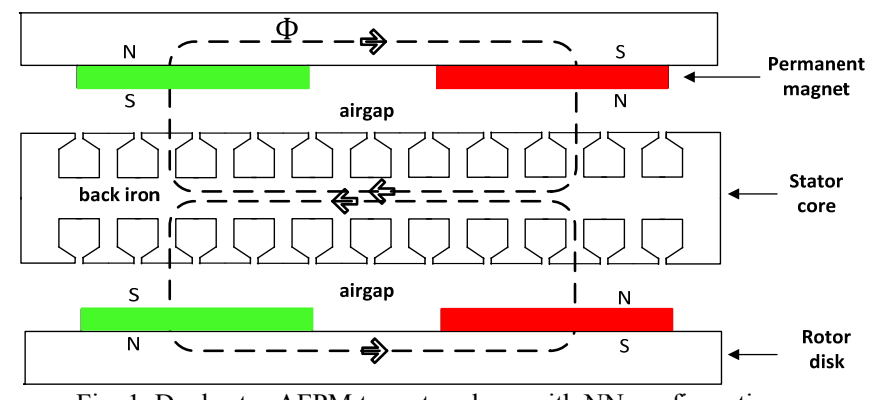

Fig. 1. Dual rotor AFPM torus topology with NN configuration.

Electric machines have a large number of design parameters. Therefore obtaining an optimized design is a challenging task because of the high number of sets of possible solutions involved. The joint use of accurate analytical sizing equations combined with the application of optimization algorithms based on artificial intelligence can help researchers and engineers to obtain an improved and faster solution of this complex problem.

Therefore, design optimization of electric machines is nowadays receiving much attention as deduced from the number of recently published papers in this topic $[9,10]$. This paper is focused to obtain an optimal solution among the huge set of possible solutions by using computationally efficient optimization algorithms leading to a global optimum solution while minimizing the computational burden.

Fault tolerance is a must in safety-critical applications such as in automotive applications. In this paper a set of analytical equations are applied to obtain the geometrical, electric and mechanical parameters of the optimized AFPM and several design restrictions are applied to ensure fault tolerance capability. Some requirements must be accomplished to design fault tolerant machines, including in electric, magnetic, physical, and thermal isolation among phases [11], so phase-tophase faults probability is minimized [12]. To this end, singlelayer fractional slot concentrated windings are particularly appropriate.

In this paper a dual outer rotor AFPM with NN configuration for automotive applications is optimized by applying accurate analytical sizing equations and taking into account fault tolerant constraints assuring a design suited for high requirements applications. To this end three constrained nonlinear optimization algorithms, which include a genetic algorithm (GA) have been applied to optimize the motor when the objective function to be optimized (maximized) is the power density.

\section{THE ANALYZED AFPM MACHINE}

The AFPM optimized in this paper is an in-wheel motor for an urban scooter. Fig. 2 shows the main machine dimensions whereas Table I shows the main motor parameters [13] as well as some of the restrictions considered in the design and optimization processes.

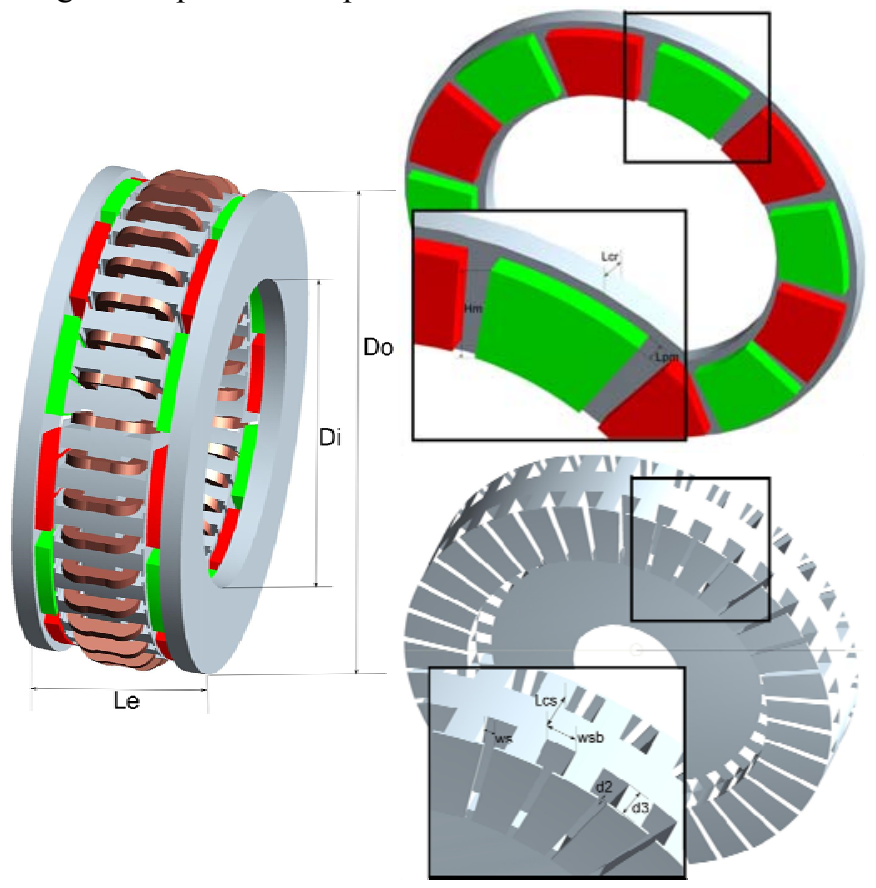

Fig. 2. Motor main dimensions. 
As shown in Fig. 2, trapezoidal magnets are used since this geometry allows minimizing the torque ripple in this kind of machines [3].

\begin{tabular}{|c|c|c|}
\hline \multicolumn{3}{|c|}{ Dimensional constraints } \\
\hline Machine outer diameter & $D_{o}$ & $<300 \mathrm{~mm}$ \\
\hline Inner to outer diameter ratio & $\lambda=D_{i} / D_{o}$ & $0.40-0.75$ \\
\hline Stator slots & $Q$ & 18 \\
\hline Motor effective axial length & $L_{e}$ & $<200 \mathrm{~mm}$ \\
\hline Air gap length & $g$ & $0.5-2.5 \mathrm{~mm}$ \\
\hline Air gap flux density & $B_{g}$ & $0.35-0.95 \mathrm{~T}$ \\
\hline \multicolumn{3}{|c|}{ Material limitation } \\
\hline $\begin{array}{l}\text { Max. stator and rotor core flux } \\
\text { densities }\end{array}$ & $B_{c s}, B_{c r}$ & $<1.5 \mathrm{~T}$ \\
\hline PM remanence & $B_{r}$ & $1.3 \mathrm{~T}$ \\
\hline PM relative permeability & $\mu_{r}$ & 1.05 \\
\hline \multicolumn{3}{|c|}{ Requirements } \\
\hline Rated line-to-line voltage & $V_{L-L}$ & $<100 \mathrm{~V}$ \\
\hline Input phase current (peak value) & $I_{s, p e a k}$ & $<20 \mathrm{~A}$ \\
\hline Number of phases & $m$ & 3 \\
\hline Number of stators & $m_{1}$ & 1 \\
\hline Output power & $P_{\text {out }}$ & $1 \mathrm{~kW}$ \\
\hline Base electrical frequency & $f_{b}$ & $50 \mathrm{~Hz}$ \\
\hline Pole pairs & $p$ & 8 \\
\hline Motor efficiency & $\eta$ & $>80 \%$ \\
\hline Electric loading & $A_{s}$ & $10-30 \mathrm{kA} / \mathrm{m}$ \\
\hline Air gap flux density & $B_{g}$ & $0.35-0.95 \mathrm{~T}$ \\
\hline Current density & $\frac{s}{J_{s}}$ & $3.5 \mathrm{~A} / \mathrm{mm}^{2}$ \\
\hline $\begin{array}{l}\text { Ratio of the average to the peak air } \\
\text { gap flux density }\end{array}$ & $\alpha_{l}$ & 0.48 \\
\hline Aspect ratio coefficient & $K_{l}$ & 0.7 \\
\hline Electrical power waveform factor & $K_{p}$ & 0.5 \\
\hline Current wave form factor & $K_{i}$ & $\sqrt{2}$ \\
\hline EMF factor & $K_{\mathrm{e}}$ & $0.692 / \eta$ \\
\hline Copper fill factor & $K_{c u}$ & 0.228 \\
\hline Carter coefficient & $K_{c}$ & 0.8 \\
\hline $\begin{array}{l}\text { Peak corrected factor of the air gap } \\
\text { flux density }\end{array}$ & $K_{f}$ & 0.8 \\
\hline Leakage flux factor & $K_{d}$ & $2 / \pi$ \\
\hline
\end{tabular}

\section{ANAlytical SizING EQUATIONS Of THE AFPM}

In this section the equations to size the AFPM are described. An analytical expression of the sizing equation for axial flux permanent magnet synchronous machines is given in [13],

$$
P_{R}=\frac{m}{m_{1}} \frac{\pi}{2} K_{e} K_{i} K_{p} K_{l} \eta B_{g} A_{s} \frac{f}{p}\left(1-\lambda^{2}\right) \frac{1+\lambda}{2} D_{o}^{2} L_{e}
$$

where the physical variables and parameters in (1) are defined in the nomenclature section.

The selection of the ratio $\lambda=D_{i} / D_{o}$ between the inner and outer diameters is critical in order to optimize machine performance, since it greatly influences the magnetic and electrical loadings. Although according to [14] the maximum value of $\lambda$ is $\frac{1}{\sqrt{3}}$, in practice $\lambda$ usually lies between $0.6-0.8$ to maximize the maximum torque to weight ratio.

The machine total outer diameter $D_{t}$ is related to the protrusion $W_{\text {сио }}$ of the end windings in the radial direction from the iron core [9] as,

$$
D_{t}=D_{o}+2 W_{\text {cuo }}
$$

Protuberances exist in both the axial and radial directions of the AFPM. The amplitude of the protrusions is related to the stator electrical loading $A_{s}$, current density $J_{S}$, and copper fill factor $K_{c u}$ as,

$$
W_{\text {cuo }}=\frac{\sqrt{D_{o}^{2}+\frac{4 A_{S} D_{g}}{K_{c u J}}}-D_{o}}{2}, \quad W_{\text {cui }}=\frac{\sqrt{D_{i}^{2}+\frac{4 A_{S} D g}{K_{c u} J_{S}}}-D_{i}}{2}
$$

The effective stack or axial length of the AFPM depends on the of rotor and stator axial lengths as,

$$
L_{e}=L_{s}+2 L_{r}+2 g
$$

The axial length of the stator is related to the stator core axial length and the amplitude of the protrusions by,

$$
L_{s}=L_{c s}+1.6 W_{\text {cui }}
$$

where the axial length of the stator core may be calculated from the magnetic flux density in the air gap $B_{g}$, the stator core flux density $B_{c S}$ and the ratio $\lambda=D_{i} / D_{o}$ as,

$$
L_{c s}=\frac{B_{g} \pi D_{o}(1+\lambda)}{B_{c s} 4 p}
$$

Similarly, the axial length of the rotor $L_{r}$ may be obtained from the axial length of rotor core $L_{c r}$ and the length of the permanent magnets $L_{P M}$ as,

$$
\begin{gathered}
L_{r}=L_{c r}+L_{P M} \\
L_{P M}=\frac{\mu_{r, P M}+B_{g}}{0.95 B_{r}-\frac{1}{K_{d}} B_{u}}\left(g+W_{c u i}\right)
\end{gathered}
$$

Note that (8) depends on the PM relative permeability and remanent flux density, $\mu_{r, P M}$ and $B_{r}$ respectively, the flux density $B_{u}$ at the surface of the PMs, and a flux leakage factor $K_{d}$.

The axial length of the rotor core $L_{c r}$ may be expressed as:

$$
L_{c r}=\frac{B_{u} \pi D_{o}(1+\lambda)}{8 B_{c r} p}
$$

$B_{c r}$ being the flux density in the rotor core.

Since the PMs are placed in the rotor discs, whereas an AC magnetic flux flows through the stator core, the flux flowing through the rotor core is almost constant.

According to [13] there is a link between the electric frequency and the stator core flux density, which may be expressed as,

$$
B_{c s}=\left\{\begin{array}{l}
5.47 f^{-0.32} \mathrm{~T} f>40 \mathrm{~Hz} \\
1.7-1.8 \mathrm{~T} \quad f \leq 40 \mathrm{~Hz}
\end{array}\right.
$$

In addition, the rotor core flux density should be within the interval,

$$
B_{c r}=1.6-1.8 \mathrm{~T}
$$

The air gap flux density in an AFPM may be expressed as:

$$
B_{g}=K_{d} B_{u}
$$

The electrical loading $A_{S}$ in AFPMs is a function of the machine average diameter $D_{g}$ :

$$
A_{s}=2 m N_{p h} \frac{I_{s, N}}{\pi D_{g}}
$$


where $D_{g}$ is calculated as,

$$
D_{g}=\frac{\left(D_{o}+D_{i}\right)}{2}
$$

The number of slots per phase and per phase and pole pair and per pole are calculated respectively as,

$$
\begin{aligned}
& q_{m p}=Q / m \\
& q_{m p}=Q / p m
\end{aligned}
$$

The number of turns per phase is calculated as,

$$
n_{s}=N_{p h} / q
$$

The air gap inductance is defined by,

$$
L_{g}=\frac{n_{S}^{2} \mu_{r, P M} \mu_{0} \theta_{c}\left(D_{o}^{2}+D_{i}^{2}\right) k_{d}}{8\left(L_{P M}+2 \mu_{r, P M} k_{c} g\right)}
$$

where $\theta_{c}=2 \pi / 2 p$ is the angular coil pitch.

The slot inductance is obtained as,

$$
L_{s}=n_{s}^{2}\left[\frac{\mu_{0} d_{3}}{3 w_{s b}}+\frac{\mu_{0} d_{2}}{0.5\left(w_{s}+w_{s b}\right)}\right]\left(\frac{D_{0}-D_{i}}{2}\right)
$$

$\omega_{s b}$ being is the slot width; $\omega_{s}$ the slot opening, $d_{2}$ the stator slot tip length and $d_{3}$ the conductor slot depth as shown in Fig. 2 .

Therefore the phase inductance is calculated as,

$$
L_{p h}=2 q_{m p}\left(L_{g}+L_{s}\right)
$$

The slot resistance is calculated from the conductor resistivity $\rho$ and the cross-section $S_{q}$ as,

$$
R_{s}=\frac{\rho n_{S}^{2}\left(D_{o}+D_{i}\right)}{2 k_{c} S_{q}}
$$

The phase resistance is calculated from the slot resistance as,

$$
R_{p h}=2 q_{m p} R_{s}
$$

The magnitude of the phase impedance results in,

$$
Z_{p h}=\left|R_{p h}+j \omega L_{p h}\right|
$$

The peak value of the electromotive force is obtained as,

$$
E M F_{\text {max }}=K_{e} N_{p h} B_{g} \frac{f}{p}\left(1-\lambda^{2}\right) D_{o}^{2}
$$

The peak value of the stator current is calculated as,

$$
I_{s, \max }=A_{s} \pi K_{i} \frac{(1+\lambda)}{2} \frac{D_{o}}{2 m N_{p h}}
$$

Finally, the power density is calculated as:

$$
P_{\text {den }}=\frac{P}{\frac{\pi}{4} D_{o}^{2} L_{e}}
$$

\section{RESTRICTIONS FOR MAXIMIZING FAULT TOLERANCE}

To ensure a high level of fault tolerance in electric machines, the phase windings should be arranged in independent modules, i.e. they should avoid as much as possible electric, magnetic, physical and thermal coupling [15, 16]. Magnetic isolation allows minimizing voltages induced in adjacent phases due to a fault current in the damaged phase. In addition, both physical and thermal isolation reduce the risk of faults between phases. Non-overlapping windings provide minimum mutual inductance between phases, thus minimizing interactions between the faulty phase and the others, so they are highly recommended in fault tolerant machines.

Restrictions imposed in this paper to ensure a high level of fault tolerance capability in the analyzed three-phase AFPM are summarized below [16],

1. Non-overlapping single-layer fractional-slot concentrated windings are used. However, because of the low winding factor of single-layer windings, $p$ should be similar but not equal to $Q$. Therefore, the following relationship between the number of stator slots $Q$ and the number of pole pairs $p$ should be accomplished: $2 p=Q \pm 2$.

2. When dealing with single-layer windings, the harmonic content of both the MMF and EMF is higher than in double-layer windings, thus increasing acoustic noise and torque pulsation levels [16]. This problem may be minimized by selecting a suitable slot/pole ratio. The number of stator teeth must be an even multiple of the number of phases to reduce acoustic noise and vibrations, thus extending the service life of the shaft bearings. This results in $Q=6 n$, where $n$ is an integer number [16]. This condition can be expressed as,

$$
Q \pm 2=2 p
$$

3. To increase the phase inductances and reduce the short circuit currents, the slots in the stator should be deep enough [12]. The phase inductance calculated as $Z_{L}=$ $E M F / I_{s}$ must be close to 1 p.u., i.e.

$$
Z_{p h}=\pi L_{b}
$$

where the base value of the phase inductance $L_{b}$ is calculated as,

$$
L_{b}=\frac{\psi_{b}}{I_{s, N}}
$$

And the base value of the flux linkage is defined as,

$$
\psi_{b}=\frac{V_{p h, N}}{\omega_{b}}
$$

$\omega_{b}=2 \pi f_{b}$ being the base electrical angular frequency.

Both the air gap and slot impedances $L_{g}$ and $L_{s}$ in (18) and (19), respectively, are adjusted during the optimization process to obtain unity phase impedance according to (28).

\section{The Optimization FunCtions DeALt With}

In this paper three optimization methods have been applied, namely genetic algorithm (GA), fmincon and fminimax solvers already implemented in the Matlab ${ }^{\circledR}$ environment.

Genetic algorithms (GAs) are a family of mathematical methods widely applied to solve optimization problems both constrained and unconstrained. GAs are search methods based on genetics and natural selection $\lceil 10\rceil$ and are being applied in the field of design optimization of electric machines $\lceil 9,14\rceil$. In the case of electrical machines design optimization, GA searches the space of the motor parameters emulating genetic reproduction mechanisms, crossover, and mutation with the objective of obtaining an optimal design. GA recurrently modifies the population of individual solutions by randomly 
selecting at each step individuals from the current population. Therefore, GA generates in at each iteration a population of individuals, the best one approaching a local optimal solution. These selected specimens are the parents which generate the children individuals for the subsequent generation. This process is repeated over successive generations with the finality of obtaining population individuals evolving toward an optimal solution. GA may be applied to solve different optimization problems which cannot be solved by traditional methods that use the gradient information of optimization since GA doesn't use the gradient information. Therefore, GA may be applied even in problems with highly nonlinear, nondifferentiable or stochastic objective functions $\lceil 17\rceil$.

In order to generate the next generation of individuals from the current population, GA uses the following rules at each step,

4. Individual's selection (parents) for the next generation.

5. Crossover operators which usually combine two parents' solutions to generate offspring or children solutions for the next generation.

6. Mutation operators which allow maintaining genetic diversity from one generation to the next. They apply random changes to individual parents and to children solutions.

Fig. 3 summarizes the steps involved in the GA method.

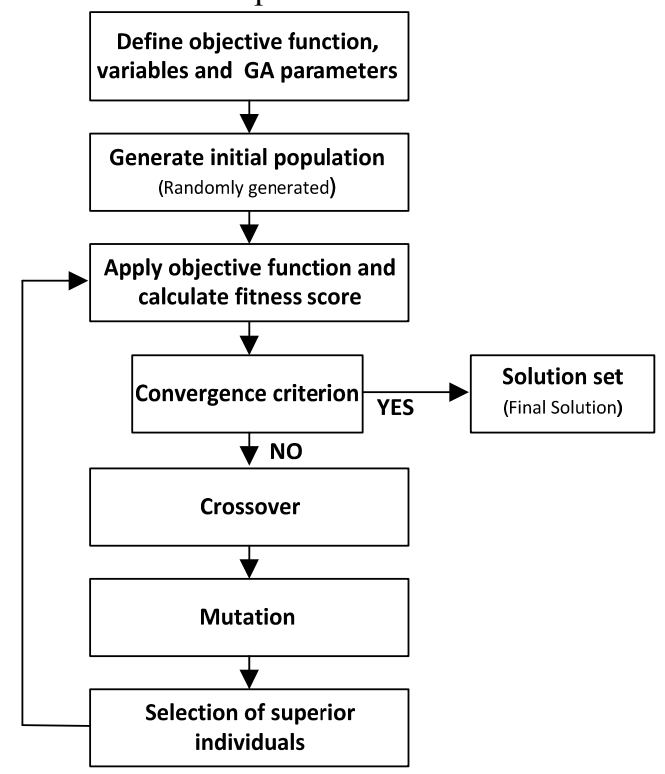

Fig. 3. Steps involved in the GA.

In machine design, there are a considerable number of free parameters. When aiming for an optimal solution, the task becomes extremely complex due to the huge number of possible combinations, unless the number of these free parameters is limited to some extent. In this paper the following eight parameters have been taken as input of the optimization algorithms: inner to outer diameter ratio, air gap flux density, air gap length, electric loading, number of turns per phase, stator slot tip length, slot opening and slot width.

In regards the coding part of the genetic algorithm, these parameters are taken as individuals, and then transformed into a binary string which will form the first chromosome.

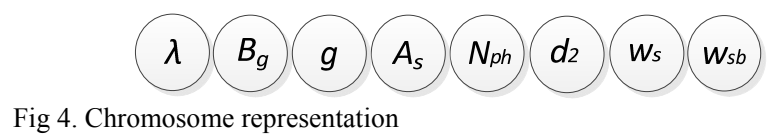

In this study, the elitist method is used as the selection operator for scattered crossover. This method of reproduction generates a random binary vector, and then chooses the genes from the first parent in which the vector is a 1 and the genes from the second parent containing a 0 , the combination of these genes forming the child.

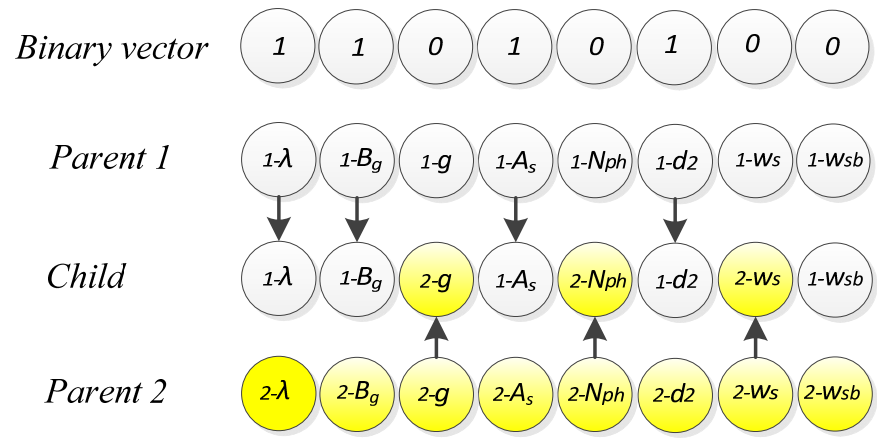

Fig 5. Scattered crossover representation.

The mutation operation randomly generates directions which are adaptive with respect to the last unsuccessful or successful generation. This process is repeated over successive generations with the finality of obtaining population individuals evolving toward an optimal solution.

Two more optimization solvers are used in this paper for comparison purposes, i.e. fmincon and fminimax. Both functions are included in the Matlab ${ }^{\circledR}$ Optimization Toolbox and can handle linear equalities and inequalities constraints, nonlinear inequality constraint functions and bound constraints.

The fmincon solver performs a constrained nonlinear minimization whereas the fminimax solver performs a minimax optimization.

Both fmincon and fminimax solvers are sequential quadratic programming (SQP) methods, i.e. nonlinear programming methods which allow solving constrained minimization problems. These quasi-Newton algorithms try to generalize the Newton's method to constrained optimization problems and are able to find local minima of constrained functions. They don't calculate the Hessian matrix, instead it is approximated by calculating different gradient vectors.

\section{RESULTS}

This section shows the results attained by applying the three aforementioned solvers, i.e. ga, fmincon and fminimax. Eight input variables which are shown in Table II are the input variables of the optimization algorithms in order to optimize the objective or fitness function, i.e. the power density.

TABLE II. INPUT VARIABLES FOR THE OPTIMIZATION ALGORITHM Input variables to optimize the fitness function

\begin{tabular}{|l|c|c|}
\hline Input optimization variables & Symbol & Bounds \\
\hline Inner to outer diameter ratio & $\lambda=D_{i} / D_{o}$ & $0.40-0.75$ \\
\hline Air gap flux density & $B_{g}$ & $0.35-0.95 \mathrm{~T}$ \\
\hline Air gap length & $g$ & $0.5-2.5 \mathrm{~mm}$ \\
\hline
\end{tabular}




\begin{tabular}{|l|c|c|} 
Electric loading & $A_{s}$ & $10-30 \mathrm{kA} / \mathrm{m}$ \\
\hline Turns per phase & $N_{p h}$ & $2-100$ \\
\hline Stator slot tip length & $d_{2}$ & $1-4 \mathrm{~mm}$ \\
\hline Slot opening & $w_{s}$ & $1-40 \mathrm{~mm}$ \\
\hline Slot width & $w_{s b}$ & $2-40 \mathrm{~mm}$ \\
\hline \multicolumn{3}{|c|}{ Variables involved in linear inequalities } \\
\hline Variables & Symbol & Inequalities \\
\hline Machine outer diameter & $D_{o}$ & $<300 \mathrm{~mm}$ \\
\hline Motor effective axial length & $L_{e}$ & $<200 \mathrm{~mm}$ \\
\hline Axial length of permanent magnets & $L_{P M}$ & $<9 \mathrm{~mm}$ \\
\hline Input phase current (peak value) & $I_{s, p e a k}$ & $<20 \mathrm{~A}$ \\
\hline
\end{tabular}

Simulation results presented in this section are compared with those provided in [18]. Results provided by this reference work were tested on laboratory. This comprehensive method allows designing an arbitrary-capacity multi-parameter doublesided AFPM motor. Table III shows the results attained the optimization process when designing a standard AFPM, i.e. when no fault tolerant restrictions are applied.

TABLE III. RESULTS ATTAINEDWHEN NO FAULT TOLERANCE RESTRICTIONS ARE CONSIDERED

\begin{tabular}{|l|c|c|c|c|}
\hline & Ref. [18] & fmincon & fminimax & ga \\
\hline$P_{\text {den }}\left[\mathrm{W} / \mathrm{cm}^{3}\right]$ & 0.360 & 0.382 & 0.382 & 0.374 \\
\hline$D_{o}[\mathrm{~m}]$ & 0.1640 & 0.1642 & 0.1642 & 0.1628 \\
\hline$N_{p h}$ & 70 & 70 & 73 & 73 \\
\hline$A_{s}[\mathrm{~A} / \mathrm{m}]$ & 15198 & 15000 & 15000 & 15100 \\
\hline$g[\mathrm{~mm}]$ & 1.1 & 1.0 & 1.0 & 1.0 \\
\hline$L_{e}[\mathrm{~mm}]$ & - & 82.6 & 82.6 & 84.5 \\
\hline$L_{P M}[\mathrm{~mm}]$ & 3.6 & 2.5 & 2.5 & 2.7 \\
\hline$L_{c r}[\mathrm{~mm}]$ & 13.0 & 13.4 & 13.4 & 13.2 \\
\hline$L_{c s}[\mathrm{~mm}]$ & 13.0 & 13.0 & 13.0 & 13.0 \\
\hline$B_{g}[\mathrm{~T}]$ & 0.48 & 0.46 & 0.46 & 0.47 \\
\hline$\lambda$ [p.u.] & 0.58 & 0.50 & 0.50 & 0.49 \\
\hline$\eta$ [p.u.] & 0.915 & 0.950 & 0.940 & 0.948 \\
\hline
\end{tabular}

Values shown in Table III show that the results attained by applying the three optimization methods are similar to those presented in [18], thus validating the approach applied.

Fig. 6 shows the evolution of the results attained by means of the three studied optimization algorithms.

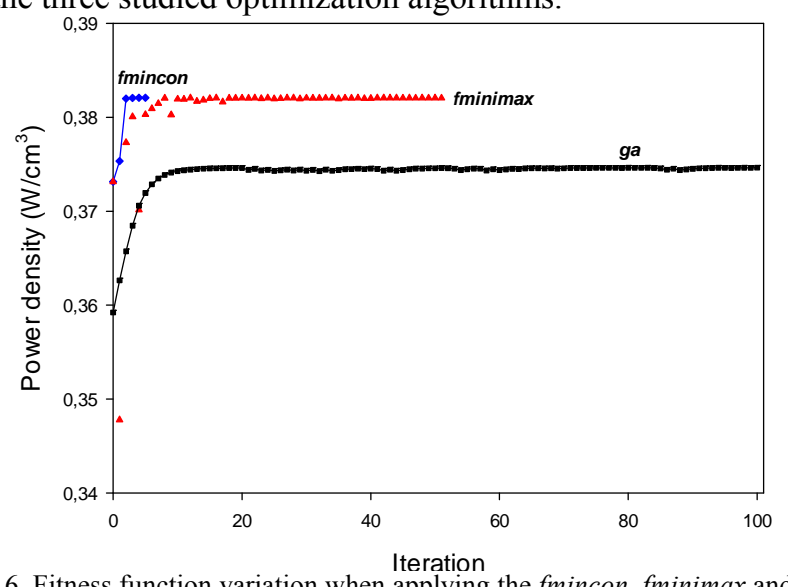

Fig. 6. Fitness function variation when applying the fmincon, fminimax and $g a$ solvers with no fault tolerant restrictions.

According to the results presented in Fig. 6, both fmincon and fminimax algorithms are faster than $\mathrm{ga}$ in attaining the optimal solution, and in this specific case they also provide a better solution (higher power density).
TABle IV. Results Attained Considering Fault Tolerance RESTRICTIONS

\begin{tabular}{|l|c|c|c|}
\hline & fmincon & fminimax & ga \\
\hline$P_{\text {den }}\left[\mathrm{W} / \mathrm{cm}^{3}\right]$ & 0.331 & 0.322 & 0.352 \\
\hline$D_{o}[\mathrm{~m}]$ & 0.1654 & 0.1672 & 0.1637 \\
\hline$N_{p h}$ & 73 & 73 & 72 \\
\hline$A_{s}[\mathrm{~A} / \mathrm{m}]$ & 15000 & 14801 & 15000 \\
\hline$g[\mathrm{~mm}]$ & 1 & 1 & 1 \\
\hline$L_{e}[\mathrm{~mm}]$ & 87.6 & 87.7 & 85.4 \\
\hline$L_{P M}[\mathrm{~mm}]$ & 1.8 & 1.6 & 2.3 \\
\hline$L_{c r}[\mathrm{~mm}]$ & 11 & 11 & 12.4 \\
\hline$L_{c s}[\mathrm{~mm}]$ & 13 & 13 & 13 \\
\hline$B_{g}[\mathrm{~T}]$ & 0.44 & 0.43 & 0.45 \\
\hline$\lambda[\mathrm{p} . \mathrm{u}]$. & 0.44 & 0.43 & 0.46 \\
\hline$\eta$ [p.u.] & 0.84 & 0.89 & 0.88 \\
\hline
\end{tabular}

By comparing the results shown in Tables III and IV, it is observed that when applying the fault tolerance restrictions the AFPM's power density unavoidably decreases by a factor less than $8 \%$ in the case analyzed. In addition the efficiency also decreases approximately by $7 \%$. In this case the $g a$ solver provides the better solution. These results show the effectiveness of the $g a$ solver in facing a nonlinear problem such as the fault tolerant design of an AFPM.

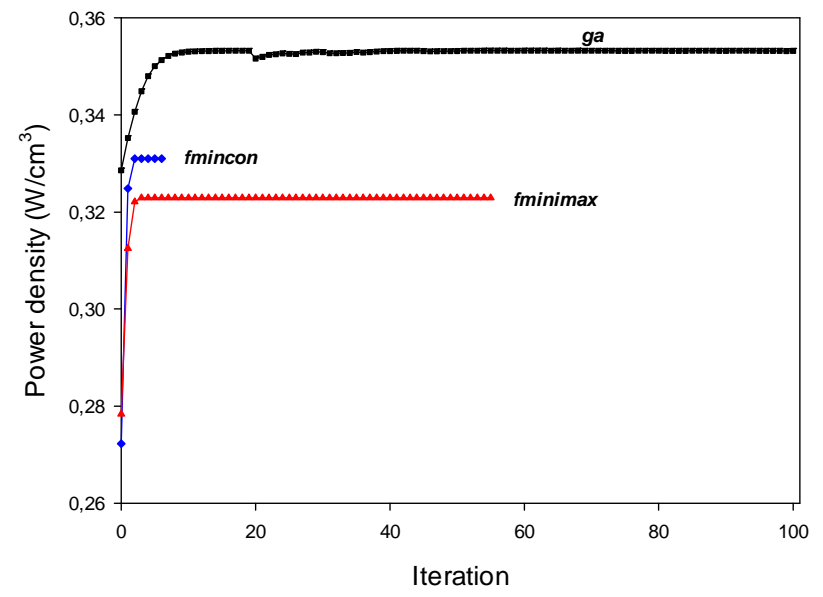

Fig. 7. Fitness function variation when applying the fmincon, fminimax and $g a$ solvers with fault tolerant restrictions.

\section{CONCLUSIONS}

In this paper an optimization process to design an in-wheel electric motor for an electric scooter has been analyzed. Concretely, a three-phase axial flux permanent magnet synchronous motor has been optimized based on in its power density by applying a set of sizing analytical equations which deal with the main geometrical, electric and mechanical parameters that define the machine. Comparisons with optimized motors data found in the technical literature show the accuracy of the applied approach since similar power densities are achieved in comparison with previous designs, even when applying fault tolerant constraints. As expected the efficiency is somewhat lowered in a fault tolerant machine mainly due to inductance constraint, because to achieve such high values of inductance an oversized stator is a must, although this could be overcome with an improved refrigeration system. In addition, fault tolerance conditions 
have been added to the optimization process in order to provide the new motor with fault tolerance capability, since it is required in safety-critical application such as the one analyzed in this paper. Results presented here shown the feasibility of the applied method to design electric motors with enhanced fault tolerance capability. In addition it has been shown that under this approach the power density of the fault tolerant optimized motor is decreased only by about $8 \%$ when compared with the reference optimized motor.

\section{REFERENCES}

[1] J.-R. Riba, A. Garcia, L. Romeral, “A Computer Experiment to Simulate the Dynamic Behavior of Electric Vehicles Driven by Switched Reluctance Motors," International Journal of Electrical Engineering Education, vol. 51, pp. X-xx, October 2014.In press

[2] K. Kamiev, J. Montonen, M. Ragavendra, J. Pyrhonen, J. Tapia, and M. Niemela, "Design principles of permanent magnet synchronous machines for parallel hybrid or traction applications," IEEE Trans. Ind. Electron., vol. 60, no. 11, pp. 4881-4890, Nov. 2013.

[3] H. Saavedra, J.-R. Riba, L. Romeral, "Magnet shape influence on the performance of AFPMM with demagnetization," IEEE Industrial Electronics Society, IECON 2013, pp.973,977, 10-13 Nov. 2013.

[4] L. Parsa, and H. A. Toliyat, "Five-Phase Permanent-Magnet Motor Drives IEEE Trans. Trans. Ind. Appl., vol. 41, no. 1, p. 30-37, Jan./Feb. 2005 .

[5] C. Attaianese, M. Di Monaco, G. Tomasso, "Multi-Source Traction Drive for Axial Flux Permanent Magnet In-Wheel Synchronous Motor," IEEE International Machines \& Drives Conference, pp. 838-843, 2011.

[6] Q.-L. Deng, F. Xiao, W.-T. Huang, "Design of New-type Axial Flux Permanent Magnet in-Wheel Machine," IEEE International Conference on Electrical and Control Engineering 2010, pp. 5831-5834, 2010.

[7] M. Aydin, S. Huang and T.A. Lipo, "Axial Flux Permanent Magnet Disc Machines: A Review", In Conf. Record of SPEEDAM, pp. 61-71, May 2004.

[8] M. Aydin, S. Huang, T.A. Lipo, "Design and 3D electromagnetic field analysis of non-slotted and slotted TORUS type axial flux surface mounted permanent magnet disc machines," Electric Machines and drives Conference, 2001. IEMDC 2001. IEEE International, pp.645651, 2001.

[9] R. Benlamine, F. Dubas, S-A. Randi, D. Lhotellier, C. Espanet, "Design by Optimization of an Axial-Flux Permanent-Magnet Synchronous Motor Using Genetic Algorithms," 2013 International Conference on Electrical Machines and Systems, Busan, Korea, pp. 13-17, Oct. 26-29, 2013.

[10] Y. Duan, D. M. Ionel, “A Review of Recent Developments in Electrical Machine Design Optimization Methods With a Permanent-Magnet Synchronous Motor Benchmark Study," IEEE Trans. Ind. Appl., vol. 49, no. 3, pp. 1268-1275, May/June 2013.

[11] A.M. El-Refaie, "Fault-tolerant permanent magnet machines: a review," IET Electr. Power Appl., vol. 5, no. 1, pp. 59-74, 2011.

[12] J.A. Haylock, B.C. Mecrow, A.G. Jack, D.J. Atkinson, "Operation of fault tolerant machines with winding failures," IEEE Trans. Energy Convers., vol. 14, no. 4, pp. 1490-1495, 1999

[13] S. Huang, J Luo, F. Leonardi, T. A. Lipo, "A Comparison of Power Density for Axial Flux Machines Based on General Purpose Sizing Equations," IEEE Trans. Energy Conv., vol. 14, vo. 2, pp. 185-192, June 1999.

[14] J. F. Gieras, R. Wang, and M. J. Kamper, Axial Flux Permanent Magnet Brushless Machines, Kluwer New York: Academic, 2004, pp. 271-350.

[15] M. A. L Badr, R. Mostafa and A.El-Wakeel, "Switched Reluctance Drive as Fault Tolerant Drive," "Proceeding of the $1^{\text {st }}$ International Conference on Electrical Engineering, ICEENG 1998, 24-26 March 1998A.

[16] P. Zheng, Y. Sui , J. Zhao , C Tong , T.A. Lipo, and A. Wang, "Investigation of a Novel Five-Phase Modular Permanent-Magnet InWheel Motor," IEEE Trans. Magn., vol. 47, no. 10, pp. 4084-4087, Oct. 2011.

[17] K Brandisky, D Sankowski and R Banasiak, I Dolapchiev, "ECT sensor optimization based on RSM and GA", COMPEL: The International Journal for Computation and Mathematics in Electrical and Electronic Engineering vol. 31, no. 3, pp. 858-869, 2012.

[18] A. Mahmoudi, S. Kahourzade, N. A. Rahim, and W. P. Hew, "Design, Analysis, and Prototyping of an Axial-Flux Permanent Magnet Motor Based on Genetic Algorithm and Finite-Element Analysis," IEEE Trans. Magn., vol. 49, no. 4, pp. 1473-1492, April 2013. 